\title{
Direct-to-consumer raw genetic data and third-party interpretation services: more burden than bargain?
}

\author{
Tia Moscarello, MS ${ }^{1}$, Brittney Murray, $\mathrm{MS}^{2}$, Chloe M. Reuter, MS ${ }^{1}$ and Erin Demo, MS ${ }^{3}$ \\ Genetics in Medicine (2019) 21:539-541; https://doi.org/10.1038/s41436-018-0097-2
}

\section{INTRODUCTION/RELEVANCE}

The latest response to consumers' increased demand for accessible genetic health information is the US Food and Drug Administration (FDA)'s recent approval of 23andMe's direct-to-consumer (DTC) genetic test for the three common $B R C A 1$ and BRCA2 pathogenic variants found in the Ashkenazi Jewish population. ${ }^{1}$ 23andMe and other DTC genetic testing companies officially report out ancestry, trait, and some health information, while also providing consumers with files of their raw genetic data. Up to $62 \%$ of consumers use third-party applications to interpret the raw data and health information not included in companies' reports. ${ }^{2}$ Little data exists on the nature and prevalence of clinical follow up of third-party raw genetic data interpretation, nor on the psychosocial impact on the consumer and their families. ${ }^{3}$ This information is of timely importance as a recent study suggests that $40 \%$ of genetic variations within DTC raw data sent for clinical confirmation are false positives. ${ }^{4}$ This raises many questions about the impact of DTC genetic testing and third-party interpretation tools, including the appropriate use of healthcare resources, clinical utility, provider and patient understanding of limitations, and psychological impact on consumers. In this commentary, we present four case vignettes from cardiovascular genetics clinics to bring awareness to possible harms of DTC raw data interpretation. Future studies investigating the breadth and frequency of cases such as these are required to understand the scope of this harm.

\section{CASES}

\section{Case 1}

A 12-year-old female presented to the aortopathy clinic for evaluation for Ehlers-Danlos syndrome type III (EDS) (OMIM 130020) because she had DTC genetic testing and the raw data report revealed a single-nucleotide polymorphism (SNP) in the COL3A1 gene (Supplementary Table 1). The patient's mother created her own database that used an internal algorithm to interpret her family's raw data.

The patient's echocardiogram and electrocardiogram (ECG) were normal. On her clinical evaluation, she did not meet clinical criteria for any types of EDS. The family requested a second opinion regarding the COL3A1 variant and were referred to medical genetics, who agreed that the patient did not meet clinical criteria for EDS. An Ehlers-Danlos, metabolic, and mitochondrial gene panel was ordered, and the reportedly pathogenic SNP in COL3A1 was not detected on clinical testing.

The patient's family continues to seek evaluations for concerns of EDS despite normal evaluations with genetics and cardiology, and negative genetic testing. The patient has seen several specialists for EDS-related concerns, including seven cardiologists, two gynecologists, an ophthalmologist, a gastroenterologist, and six emergency room physicians for concerns of syncope. Four other relatives have undergone evaluations by a geneticist for EDS, the results of which the patient's mother would not share.

\section{Case 2}

A 36-year-old male with idiopathic recurrent myocarditis was referred for genetic counseling after he shared an interpretation report of his DTC raw genetic data with his general cardiologist. The report indicated the patient carried a SNP that corresponded to a pathogenic variant in $M Y B P C 3$ (NM_000256.3:p.Asp770Asn; rs36211723), a gene associated with hypertrophic cardiomyopathy (HCM) (Supplementary Table 1).

The patient arrived eager to understand how this variant could be related to his myocarditis, unaware of its association with HCM. Review of clinical cardiovascular notes, imaging, and family history were not suggestive of a diagnosis of HCM. Clinical genetic testing of the MYBPC3 gene was sent. Pretest counseling included discussion of the possibility that the $M Y B P C 3$ variant may not be clinically confirmed and that,

\footnotetext{
${ }^{1}$ Stanford Center for Inherited Cardiovascular Disease, Stanford Health Care, Stanford, California, USA; ${ }^{2}$ Johns Hopkins University Department of Medicine, Division of Cardiology, Baltimore, Maryland, USA; ${ }^{3}$ Sibley Heart Center Cardiology, Children’s Healthcare of Atlanta, Atlanta, Georgia, USA. Correspondence: Erin Demo demoe@kidsheart. com)
} 
because he did not carry a diagnosis of HCM, his insurance may not cover the cost of the clinical genetic testing. The patient expressed that he was "crossing his fingers" that the variant was a false positive. The MYBPC3 variant was not detected in clinical genetic testing and the patient was relieved. Ongoing cardiac screening for HCM (in patient or his family) was not recommended.

\section{Case 3}

A healthy 22-year-old male referred himself to the HCM clinic for an evaluation after discovering the same $M Y B P C 3$ SNP as case 2 (NM_000256.3:p.Asp770Asn; rs36211723) (Supplementary Table 1) in his raw genetic data obtained from DTC genetic testing. During the clinical intake, the patient had significant anxiety about this result: he had taken medical leave from his $\mathrm{PhD}$ program after learning of his results "to focus on [my] HCM and risk of sudden death." He was an avid cyclist, but gave it up after learning that vigorous exercise is not recommended for people with HCM. He had looked into joining a support group, but did not feel ready to discuss the possibility of myectomy or transplant "yet."

In the appointment, the patient described that he was not surprised by the results; he attributed his history of palpitations during childhood and his father's left ventricular hypertrophy (LVH) to HCM. After reviewing the patient's echocardiogram and ECG and his father's records, the cardiologist assessed that there was no clinical evidence for HCM in the patient or his father. Confirmatory testing of the DTC results confirmed that this SNP was not detected. In calling the patient with these results, he was emotional and relieved. This patient was released from screening for HCM.

\section{Case 4}

An 18-year-old female died suddenly while running. Her autopsy was inconclusive. Prior to her death, the proband and her father had submitted DTC samples, and this prompted him to research the raw data. The raw data interpretation identified multiple SNPs classified as pathogenic for arrhythmogenic right ventricular cardiomyopathy (ARVC) (Supplementary Table 1). Therefore, the proband's 15-year-old sister was screened for ARVC, and cardiac magnetic resonance imaging (cMRI) showed an aneurysm in the apex of the RV. An implantable cardioverter defibrillator (ICD) was placed for primary prevention. Clinical genetic testing later revealed that the sister did not carry the PKP2 variant found on DTC testing. Concerned, the cardiologist referred the family to a specialty center. Upon expert evaluation, the sister's cMRI was found to be normal and she did not meet diagnostic criteria for ARVC. The remaining autopsy sample was obtained and clinical genetic testing for arrhythmia and cardiomyopathy genes, including $P K P 2$, did not identify the variant found by the DTC genetic testing company. Given that the benefits of an ICD in an asymptomatic individual do not outweigh risks of the device, the sister's device was explanted. The family remains concerned and confused about the cause of their daughter's death, and of their surviving daughter's risk.

\section{THEMES}

Consumers are motivated to access their raw genetic data to learn more about their personal health through third-party interpretation tools and will sometimes undergo rigorous and unnecessary clinical evaluations to follow up on health information obtained through raw genetic data. These cases are examples of analytical false positives: they were "detected" in a genotyping SNP array performed at a DTC testing company, released as raw data, and typed into a third-party interpretation tool that suggested pathogenicity. Patient and nongenetics provider misunderstandings may arise, perpetuating inaccurate genetic risk assessment, which could result in medical mismanagement. Some patients experience heightened anxiety as a result of the confusing and often misleading interpretation.

\section{CALL TO ACTION}

As the demand for DTC genetic testing grows and the availability of raw data interpretation tools remain available, we anticipate the consumer themes illustrated above are likely to increase. While DTC genetic testing can empower patients to learn about some medically actionable genetic risk, there are also clear limitations of and misconceptions surrounding such testing.

The medical utility of DTC genetic testing is low, but even our limited experience demonstrates that it is placing a burden on the healthcare system to (1) clinically confirm these genetic results and (2) confirm that a patient does not have a clinical diagnosis of the disease associated with the reported SNP. The recommendation that all results obtained from the raw genetic data of DTC tests need to be clinically confirmed ${ }^{5}$ and recent data on false-positive rates indicate that almost half of consumers seeking clinical confirmation of their genetic testing results would be a waste of healthcare dollars. ${ }^{4}$

There is limited research on what consumers do with the interpretation of their raw genetic data. Recent studies suggest that $20-30 \%$ of consumers share DTC genetic testing results, including raw data interpretation with one or more healthcare providers. ${ }^{2,}{ }^{6}$ However, despite its clear medical importance given the propensity for false positives, it is not known how many consumers seek a clinical evaluation for the conditions associated with the identified $\mathrm{SNP}(\mathrm{s})$, or clinical confirmation of the identified variants. It is also unclear how many healthcare providers sustain misconceptions about the validity of these results. To better understand the prevalence and extent of such outcomes, more research is needed to understand the patient experience and possible harms of receiving raw data interpretation results. To inform policy, future studies should investigate the frequency of these outcomes. Both the clinical and consumer genetics communities must collaborate to ensure consumers have access to valid data and correct interpretations of it. Studies exploring the education, support, and medical care surrounding DTC genetic testing and third-party raw data interpretation are needed to minimize harms to patients and reduce unnecessary costs to the healthcare system. 


\section{ELECTRONIC SUPPLEMENTARY MATERIAL}

The online version of this article (https://doi.org/10.1038/s41436018-0097-2) contains supplementary material, which is available to authorized users.

\section{DISCLOSURE}

The authors declare no conflict of interest.

\section{REFERENCES}

1. US Food and Drug Administration. FDA authorizes, with special controls, direct-to-consumer test that reports three mutations in the BRCA breast cancer genes. https://www.fda.gov/NewsEvents/Newsroom/ PressAnnouncements/ucm599560.htm. March 6, 2018.needs an access date

2. Wang C, Cahill TJ, Parlato A2, Wertz B, Zhong Q, Cunningham TN, Cummings JJ. Consumer use and response to online third-party raw DNA interpretation services. Mol Genet Genomic Med. 2018;6:35-43.

3. Badalato L, Kalokairinou L, Borry P. Third party interpretation of raw genetic data: an ethical exploration. Eur J Hum Genet. 2017;25: 1189-1194.

4. Tandy-Connor, S, Guiltinan, J, Krempely $\mathrm{K}$ et al. False-positive results released by direct-to-consumer genetic tests highlight the importance of clinical confirmation testing for appropriate patient care. Genet Med 2018;1-7.
5. 23andMe Reports Included in Service Access Date: 4/12/2018. https:// www.23andme.com/dna-reports-list/

6. Stewart KFJ, Wesselius A, Schreurs MAC, et al. Behavioural changes, sharing behaviour and psychological responses after receiving direct-toconsumer genetic test results: a systematic review and meta-analysis. J Community Genet. 2018;9:1-18.

Open Access This article is licensed under a Creative Commons Attribution-NonCommercial-ShareAlike 4.0 International License, which permits any non-commercial use, sharing, adaptation, distribution and reproduction in any medium or format, as long as you give appropriate credit to the original author(s) and the source, provide a link to the Creative Commons license, and indicate if changes were made. If you remix, transform, or build upon this article or a part thereof, you must distribute your contributions under the same license as the original. The images or other third party material in this article are included in the article's Creative Commons license, unless indicated otherwise in a credit line to the material. If material is not included in the article's Creative Commons license and your intended use is not permitted by statutory regulation or exceeds the permitted use, you will need to obtain permission directly from the copyright holder. To view a copy of this license, visit http://creativecommons.org/licenses/by-nc-sa/4.0/.

(C) The Author(s) 2018 